\title{
The Prevalence and Predictors of Depressive, Anxiety, and Stress Symptoms Among Tepi Town Residents During the COVID-19 Pandemic Lockdown in Ethiopia
}

\author{
Seid Ali Tareke ${ }^{1}$ - Mesfin Esayas Lelisho ${ }^{1}$ (1) - Sali Suleman Hassen ${ }^{1}$ - Adem Aragaw Seid ${ }^{1} \cdot$ Sebwedin Surur Jemal $^{1}$. \\ Belete Mulatu Teshale ${ }^{1} \cdot$ Teramaj Wongel Wotale $^{2} \cdot$ Binay Kumar Pandey $^{3}$
}

Received: 6 October 2021 / Revised: 21 November 2021 / Accepted: 22 November 2021 / Published online: 14 January 2022

(c) W. Montague Cobb-NMA Health Institute 2021

\begin{abstract}
Background As a result of the coronavirus disease 2019 (COVID-19) outbreak, many countries have imposed movement restrictions and implemented lockdowns. However, evidence from a variety of nations showed that the COVID-19 outbreak and its associated quarantine measures triggered a wide range of psychological problems, such as anxiety, depression, and stress in the general population. As a result, the purpose of this study was to determine the prevalence and predictors of depression, anxiety, and stress symptoms among Tepi town residents during the pandemic lockdown.

Methodology A community-based cross-sectional survey was conducted among residents of Tepi town from September 15 through September 25, 2020, and residents who have lived in Tepi town for at least 6 months were included. We have employed the depression, anxiety, and stress scale 21 (DASS-21) to evaluate depression, anxiety, and stress. The Chi-squared test of association and logistic regression were used to identify factors associated with depression, anxiety, and stress among residents of Tepi town. For all statistical analysis, we used (IBM) SPSS version 25.

Results According to the current study, the prevalence of depression, anxiety, and stress symptoms were $37.7 \%, 39.0 \%$, and $44.2 \%$, respectively, among residents of Tepi town. Estimated odds of having depression, anxiety, and stress were as follows: for being female 6.315, 4.591, and 3.155; smoking 1.787, 1.883, and 1.787; sleep problem 2.613, 2.254, and 1.721; chewing Khat 2.156, 2.053, and 2.110; quarantine for 14 days 2.251, 1.902, and 1.960; and frequent use of social media $3.126,1.849$, and 3.126 times more likely as compared to their corresponding reference group respectively. The odds of developing depression and anxiety respectively were as follows: for alcohol consumption 2.438 and 1.797 times higher than their corresponding reference group respectively. Those exposed to COVID-19 were 3.870 times more likely to develop depression symptoms. Estimated odds of having anxiety and stress symptoms for fear of COVID-19 were 1.776 and 1.835; social interactions altered were 3.197 and 2.069 , moderate levels of hope were 2.687 and 2.849 respectively. The odds ratio for those taking traditional preventive medicine, and having family members infected with COVID-19 were 2.475 and 1.837 times more likely to experience anxiety symptoms respectively.

Conclusion In this study, the prevalence of depression, anxiety, and stress symptoms was found to be high among residences in Tepi town. Being female, chewing Khat, smoking, being quarantined for 14 days, frequently using social media, and having sleeping problems were all found to be significantly associated with an increased risk of developing depression, anxiety, and stress symptoms, whereas alcohol consumption and family members infected by COVID-19 were considerably linked to depression and anxiety symptoms. Fear of COVID-19, influence on social interaction and having a moderate level of hope were substantially related to stress and anxiety symptoms, while taking preventive medicine was found to be a significant factor in anxiety symptoms among Tepi town residences. Interventions should be made to improve the mental health of Tepi residents.
\end{abstract}

Keywords Depression · Anxiety · Stress · COVID-19 - Lockdown

Mesfin Esayas Lelisho

mesfinstat27@gmail.com

Extended author information available on the last page of the article 


\section{Introduction}

The coronavirus disease 2019 (COVID-19) outbreak began in Wuhan, China, and has rapidly expanded to other nations throughout the world [1]. On January 30, 2020, the World Health Organization declared it to be a global public health disaster and on March 11, 2020, a pandemic [2]. Then, countries have implemented containment measures such as contact limitations, self-isolation, school and college/university closures, quarantine, and social distancing [3]. Many countries, including Ethiopia, imposed nationwide lockdown measures, resulting in profound social and cultural changes.

Because COVID-19 emerges suddenly and is extremely infectious, it poses major risks to people's physical health and lives $[1,4,5]$. Mental health professionals believe that the pandemic and the resulting lockdown influenced the mental health of the global population, with an increase in cases of insomnia, sadness, and self-harm [6]. In line with this, data from various countries showed that the COVID-19 outbreak and associated quarantine measures hurt mental health and resulted in a wide range of psychological issues, such as anxiety, depression, suicidal ideation, and stress in the general population $[1,7,8]$. According to recent research reports [9-11], elevated levels of stress, anger, anxiety, and depression have been reported among individuals in various parts of the world during the COVID-19 pandemic.

People's fear of the virus' unknown nature can contribute to a high prevalence of mental diseases [12]. According to the recent meta-analysis done on the general population, the prevalence of depression, anxiety, and stress during the COVID-19 pandemic was $33.7 \%, 31.9 \%$, and $29.6 \%$, respectively [13]. A similar study from Indonesia reported a prevalence of depression of $10.15 \%$, anxiety of $9.25 \%$ and stress of $2.39 \%$ [14]. In the early stages of the COVID-19 pandemic, at Gondar, Ethiopia, the prevalence of depression, anxiety, and stress was $32.0 \%, 25.8 \%$, and $14.7 \%$, respectively [15].

Other study findings indicated that the prevalence of psychological disorders was significantly higher. A study in Spain found that $36 \%$ of study participants had moderate to severe psychological issues, with $25 \%$ having mild to severe anxiety, $41 \%$ having depression, and $41 \%$ having stress symptoms [16]. Furthermore, depression, anxiety, and stress were found to be prevalent in $39.0 \%, 47.0 \%$, and $35.8 \%$ of the European population, respectively [17]. According to another comprehensive review analysis, the prevalence of depression, anxiety, and stress was $20 \%$, $35 \%$, and $53 \%$, respectively [18].

According to studies done on psychological disorders during the COVID-19 pandemic, those who were afflicted had a variety of mental trauma symptoms, including emotional distress, depression, stress, irritability, insomnia, and anger [7, 19, 20]. In addition, a higher frequency of psychological distress symptoms such as sadness and anxiety was related to younger age groups and females [21]. Social-economic position, leisure activity, sex, age, monthly income, and perceived wellbeing are all variables associated with mental health outcomes [16, 22]. Studies in Italy have demonstrated an increased incidence of psychological distress and post-traumatic stress symptoms due to increased numbers of days locked up, living alone while at a lock, smoking, and lengthy duration of disease during the acute phase of the COVID-19 pandemic [22-24].

Depression, anxiety, and stress all have an impact on the outcome of chronic diseases like diabetes, cardiovascular disease, cancer, and obesity [25]. Furthermore, poor mental health is linked to inactivity, interpersonal connection issues, suicidal ideation, and sleeplessness problems [26]. So, it is vital to investigate its prevalence and implement interventions to alleviate its symptoms in these populations. However, research on the mental health of people in underdeveloped nations like Ethiopia is scarce. Therefore, the main goal of this study was to determine the prevalence and associated factors of depression, anxiety, and stress symptoms among the residents of Tepi town. The insights from this study could be used by policymakers, healthcare planners, medical professionals, and other concerned bodies to design prevention strategies for depression, anxiety, and stress symptoms related to COVID 19.

\section{Material and Methods}

\section{Study Design and Setting}

A community-based cross-sectional study was conducted on all permanent residents in Tepi Town, Southwestern Ethiopia from September 15 to September 25, 2020. Residents who lived in Tepi town for at least 6 months were included in the study. However, those who lived for less than six months in Tepi town and those who were not able to give the required information were excluded from the study.

\section{Sampling Procedure}

The sample size was calculated using a single population proportion formula and a simple random sampling technique [27]. The number of samples required for the study was estimated by taking $50 \%$ of the prevalence of depression, anxiety, and stress among residents in a single population proportion for unknown prevalence, with $5 \%$ of marginal error and $10 \%$ non-response rate. Then the sample size for this study becomes $384.5+39 \approx 424$. However, 24 
questionnaires were discarded due to missing information or refusal to reply, resulting in 400 participants in the study.

\section{Study Variables, Data Collection Tools, and Measurements}

The response variable was depression, anxiety, and stress symptoms $\left(Y_{i}, W_{i}\right.$, and $\left._{i}\right)$, which is dichotomized as

$Y_{i}=\left\{\begin{array}{c}1, \text { presenceofdepressionsymptoms } \\ 0, \text { otherwise }\end{array}\right.$

$W_{i}=\left\{\begin{array}{c}1, \text { presenceofAnxietysymptoms } \\ 0, \text { otherwise }\end{array}\right.$

$Z_{i}=\left\{\begin{array}{c}1, \text { presenceofStresssymptoms } \\ 0, \text { otherwise }\end{array}\right.$

To assess depression, anxiety, and stress, we used the depression, anxiety, and stress scale-21 (DASS-21) [28]. The DASS-21 provides a short and concise measure of a mental health problem and has already been validated using the Indonesian language [29]. It consisted of 21 items: seven items for anxiety (items 2, 4, 7, 9, 15, 19, and 20), seven items for depression (items 3, 5, 10, 13, 16, 17, and 21), and seven items for stress (items 1, 6, 8, 11, 12, 14, and 18). Each item was measured on a 4-point Likert scale, ranging from 0 "never" to 3 "always." The total score of each construct (consisting of seven items) was multiplied by two to determine the amount of depression, anxiety, and stress. For this study, we have used dichotomized categories as considered present if the sum of the scores on the corresponding DASS items $\geq 10, \geq 8$, and $\geq 15$ for depression, anxiety, and stress, respectively [30]. The reliability (Cronbach's alpha $=0.87$ ) and construct validity of the Arabic version of DASS-21 are well confirmed.

The PSQI is a 19-item, self-rated questionnaire designed to measure sleep quality and disturbance over the past month in clinical populations [31], which has been used elsewhere [32-35]. The 19 items are grouped into 7 components, including (1) sleep duration, (2) sleep disturbance, (3) sleep latency, (4) daytime dysfunction due to sleepiness, (5) sleep efficiency, (6) overall sleep quality, and (7) sleep medication use. Each of the sleep components yields a score ranging from 0 to 3, with 3 indicating the greatest dysfunction. The sleep component scores are summed up to yield a total score ranging from 0 to 21 , with the higher total score (referred to as the "global score") indicating worse sleep quality. A global PSQI score of 5 or higher indicates good sleep quality with no sleep problems. The hearth hope index (HHI) was chosen to evaluate the level of hope. The HHI contains 12 items, with each item scoring from 1 (strongly disagree) to 4 (strongly agree). Then the total score is $12-48$. A total score from 12 to 23 indicates a low level of hope, 24 to 35 as a moderate level, and 36 to 48 as a high level [36, 37]. In this study, we checked internal consistency and reliability, and hence Cronbach's alpha for HHI was 0.901.

Explanatory variables consist of demographic variables (gender), substance use (alcohol, Khat, and smoking habits), and COVID-19-related perceptions (stayed in quarantine for 14 days, fear of COVID-19, frequent use of social media, influence on social interaction, taking preventive medicine, sleep problems, family members infected by COVID-19, exposed to COVID-19 Infection). In addition to this, the Hearth Hope Index was used as the independent variable.

\section{Method of Data Analysis}

To highlight descriptive results, we used frequency distribution and percentages. The Chi-squared test of association was employed to examine the relationship between response and explanatory variables. The logistic regression model was utilized to find determinants of depression, anxiety, and stress symptoms. Multivariable logistic regressions were conducted by taking all significant covariates in the univariable analysis at a significance level of $25 \%$ [38]. We used SPSS version 25 to perform all statistical analyses.

\section{Statistical Models}

\section{Logistic Regression}

Logistic regression analysis studies the association between a categorical dependent variable and a set of independent (explanatory) variables.

\section{Binary Logistic Regression}

When the dependent variable is dichotomous, such as the existence or absence of a certain event, binary logistic regression is utilized, and the independent variables can be of any type. For the Bernoulli trial, the Bernoulli distribution defines probabilities $\mathrm{P}(\mathrm{Y}=1)=\pi$ and $\mathrm{P}(\mathrm{Y}=0)=1-\pi$, with $\mathrm{E}(\mathrm{Y})=\pi$.

The general model for binary logistic regression is as follows:

$\log i t(\pi(x i))=\log \left(\frac{\pi(x i)}{1-\pi(x i)}\right)=\beta o+\beta_{1} X_{1}+\beta_{2} X_{2}+\ldots .+\beta_{K} X_{k}$

where $x_{i}$ is an explanatory variable in the model, $\pi$ is the probability of success, $1-\pi$ is the probability of failure, $\beta_{0}$ is constant terms/intercept, and $\beta_{i}$ is the coefficients or slope of the explanatory variable in the model. 


\section{Parameter Estimation}

The maximum likelihood estimation (MLE) approach maximizes a likelihood function to estimate the parameters of a probability distribution [39].

The maximum likelihood estimates of the parameters could be obtained by maximizing the log-likelihood function form given by

$\pi(x i)=\frac{\exp \left(\beta o+\beta_{1} X_{1}+\beta_{2} X_{2}+\ldots .+\beta_{K} X_{k}\right)}{1+\exp \left(\beta o+\beta_{1} X_{1}+\beta_{2} X_{2}+\ldots .+\beta_{K} X_{k}\right)}$

Since observing values of $Y$ say, $Y_{i}^{\prime} s(i=1,2 \ldots n)$ are independently distributed as Bernoulli, the maximum likelihood function of $\mathrm{Y}$ is given by

$L(\beta / y)=\prod_{i=1}^{n} P(y i / x i)=\prod_{i=1}^{n}\left[\frac{e^{x_{i} \beta_{i}}}{1+e^{x_{i} \beta_{i}}}\right]^{y i}=\left[\frac{1}{1+e^{x_{i} \beta_{i}}}\right]^{\left(1-y_{i}\right)}$

\section{The Wald Test Statistic}

The Wald test is a way of testing the significance of particular explanatory variables in a statistical model [40]. Wald $\chi^{2}$ statistics are used to test the significance of individual coefficients in the model and are calculated as follows:

$W=\left(\frac{\beta}{\operatorname{se}(\beta)}\right)^{2} \sim x_{(1)}^{2}$

where $s e(\beta)$ represents the standard error of the regression coefficient $\beta$. $W$ assumes a Chi-squared distribution with one degree of freedom.

The maximum likelihood estimate of the parameter is found by the derivation of the log-likelihood function to each $\beta$ 'sand set each equation to zero, which is given as $\mathrm{j}=1,2, \ldots, \mathrm{k}$

$\frac{d \log L(\beta / y)}{d \beta_{j}}=0, j=1,2, \ldots, k$

\section{Model Adequacy Checking}

The Hosmer and Lemeshow test is a popular method for determining the model's goodness of fit, and it allows for any number of explanatory variables [40].

The Hosmer and Lemeshow test statistics are defined as

$C^{2}=\sum_{i=1}^{p}\left[\frac{\left(O_{i}-E_{i}\right)^{2}}{m_{i} p_{i}\left(1-p_{i}\right)}\right]$ where $O_{i}, E_{i}, m_{i}, p_{i}$ denote the observed events, expected, observations, and the average predicted risk for the $i^{\text {th }}$ risk desire group respectively.

The statistical hypothesis of the Hosmer and Lemeshow test is as follows:

$\mathrm{H}_{1}$ : The model is good to fit the data well.

$\mathrm{H}_{0}$ : The model is not good enough to fit the data.

The decision rule is that we do not reject the null hypothesis if the $P$ value $>\alpha$ value $=0.05$.

\section{Results}

The purpose of this study was to determine the prevalence and associated factors of depression, anxiety, and stress symptoms among Tepi town residents during the COVID19 pandemic lockdown. To investigate the prevalence and determinants of depression, anxiety, and stress symptoms, descriptive and inferential analyses were employed.

\section{Demographic Variables and Substance Use of the Participants}

The high percentage of participants in this study, 269 (67.2\%) of the 400 citizens of Tepi town, were females. Alcoholism, Khat chewing, and smoking were practiced by approximately $41.0 \%, 35.5 \%$, and $37.5 \%$ of participants, respectively (Table 1 ).

\section{Participants' Perception Characteristics Related to COVID-19}

It is also shown that, in terms of the impact of social interaction, the majority of participants $(61.0 \%)$ reported difficulties. More than half of the participants $(54.0 \%)$ used social media frequently, and $50.5 \%$ were concerned about the COVID-19 pandemic. More than one-third of the participants (33.3\%) had previously spent 14 days in quarantine. Nearly two-thirds $(63.0 \%)$ of the participants had a moderate level of hope (Table 1).

\section{COVID-19 Related Behavior of the Participants}

More than half of the participants, 224 (56.0\%), attempted to take traditional preventive medicine in the hope of preventing COVID-19. The majority of the participant's family members, 229 (57.2\%), were exposed to COVID-19 infection. COVID-19 infected $32.8 \%$ of participants' family members. In total, $55.2 \%$ of the participants had sleeping issues (Table 1). 
Table 1 Descriptive summaries and Chi-squared test association between depression, anxiety, and stress with explanatory variables

Fig. 1 Prevalence of depression, anxiety, and stress among Tepi town residents, Southwest Ethiopia

\begin{tabular}{|c|c|c|c|c|c|}
\hline \multirow[t]{2}{*}{ Variables } & \multirow[t]{2}{*}{ Categories } & \multirow[t]{2}{*}{$N(\%)$} & \multicolumn{3}{|l|}{$P$ value $^{\mathrm{a}}$} \\
\hline & & & Depression & Anxiety & Stress \\
\hline \multirow[t]{2}{*}{ Gender } & Male & $131(32.8)$ & 0.006 & $<0.001$ & 0.022 \\
\hline & Female & $269(67.2)$ & & & \\
\hline \multirow[t]{2}{*}{ Alcohol habit } & No & $236(59.0)$ & $<0.001$ & 0.018 & 0.002 \\
\hline & Yes & $164(41.0)$ & & & \\
\hline \multirow[t]{2}{*}{ Khat chewing habit } & No & $258(64.5)$ & 0.001 & 0.019 & 0.002 \\
\hline & Yes & $142(35.5)$ & & & \\
\hline \multirow[t]{2}{*}{ Smoking habit } & No & $250(62.5)$ & 0.027 & $<0.001$ & 0.026 \\
\hline & Yes & $150(37.5)$ & & & \\
\hline \multirow{2}{*}{$\begin{array}{l}\text { Stayed in quarantine } \\
\text { for } 14 \text { days }\end{array}$} & No & $268(67.0)$ & $<0.001$ & 0.007 & 0.006 \\
\hline & Yes & $132(33.0)$ & & & \\
\hline \multirow[t]{2}{*}{ Frequent use of social media } & No & $184(46.0)$ & $<0.001$ & 0.355 & 0.035 \\
\hline & Yes & $216(54.0)$ & & & \\
\hline \multirow[t]{2}{*}{ Fear of COVID-19 } & No & $198(49.5)$ & 0.001 & 0.053 & $<0.001$ \\
\hline & Yes & $202(50.5)$ & & & \\
\hline \multirow[t]{2}{*}{ Influence on social interaction } & No & $156(39.0)$ & 0.280 & 0.213 & 0.013 \\
\hline & Yes & $244(61.0)$ & & & \\
\hline \multirow[t]{2}{*}{ Taking preventive medicine } & No & $176(44.0)$ & 0.356 & 0.163 & 0.002 \\
\hline & Yes & $224(56.0)$ & & & \\
\hline \multirow[t]{2}{*}{ Sleep problems } & No & $179(44.8)$ & $<0.001$ & $<0.001$ & $<0.001$ \\
\hline & Yes & $221(55.2)$ & & & \\
\hline \multirow[t]{2}{*}{ Family member infected by COVID-19 } & No & $269(67.2)$ & $<0.001$ & 0.053 & $<0.001$ \\
\hline & Yes & $131(32.8)$ & & & \\
\hline \multirow{2}{*}{$\begin{array}{l}\text { Exposed to COVID-19 } \\
\text { Infection }\end{array}$} & No & $171(42.8)$ & 0.009 & 0.734 & 0.005 \\
\hline & Yes & $229(57.2)$ & & & \\
\hline \multirow[t]{3}{*}{ Hearth hope index } & Low level & $127(31.8)$ & 0.008 & $<0.001$ & $<0.001$ \\
\hline & Moderate level & $252(63.0)$ & & & \\
\hline & High level & $21(5.2)$ & & & \\
\hline
\end{tabular}

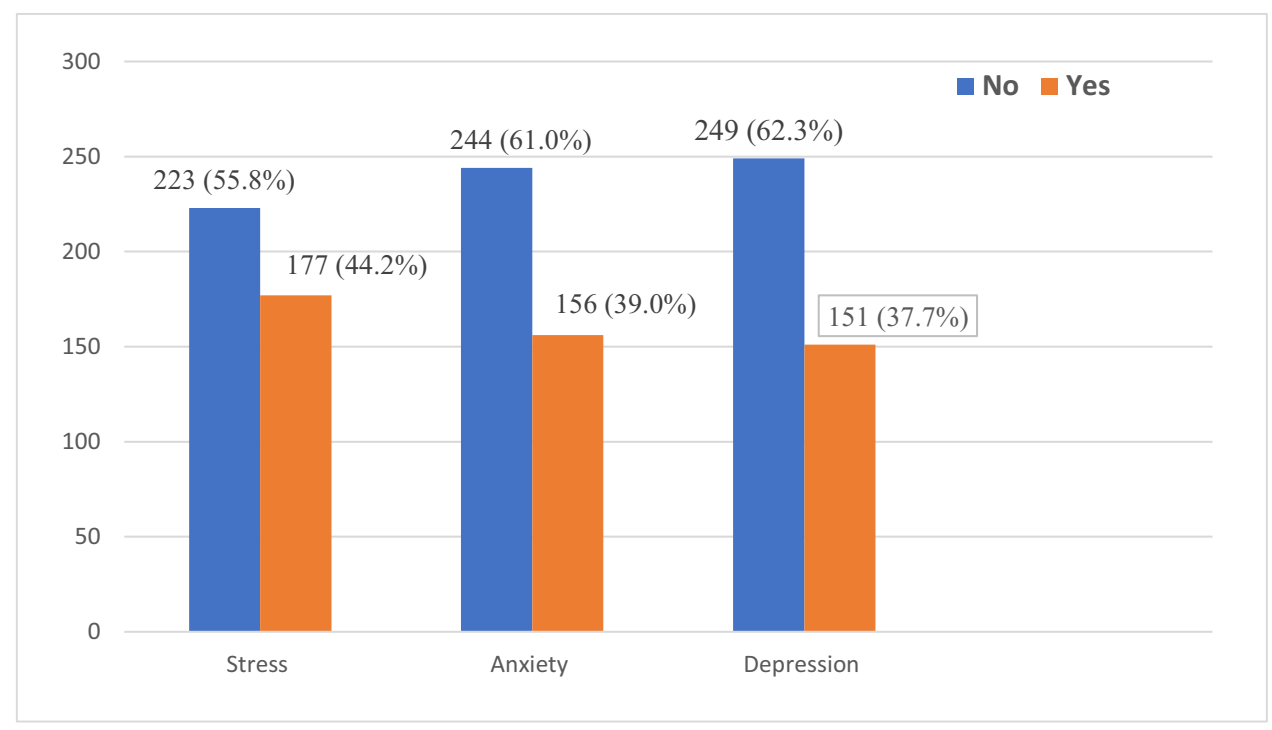


The prevalence of depression, anxiety, and stress among Tepi residents was $151(37.7 \%), 156(39.0 \%)$, and 177 (44.2\%), respectively (Fig. 1).

\section{Multivariable Binary Logistic Regression Results}

We have fitted three models for the three response variables, i.e., depression, anxiety, and stress. For each response, a separate binary logistic regression was fitted using a variable significant at a $25 \%$ level of significance at a univariable level. Accordingly, the results for each model are presented in the following sections.

\section{Factors Associated with Depressive Symptoms among Tepi Town residents}

According to multivariable binary logistic analysis, being female, alcohol consumption, chewing Khat, smoking, being quarantined for 14 days, frequent use of social media, sleep problems, family members infected by COVID-19, and exposure to COVID-19 were found to be significant factors in depressive symptoms among residents of Tepi town, whereas fear of COVID-19 and HHI were not significant variables at $5 \%$ level of significance.

Female respondents were 6.315 times [95\% CI: 2.778-14.357] more likely than males to experience the depression symptoms. When compared to respondents who did not consume alcohol, those who had a history of drinking had 2.438 times [95\% CI: 1.457-4.078] more likely to develop depression symptoms. Moreover, smokers had a 1.787 [95\% CI: 1.074-2.972] times higher likelihood of having depression than non-smokers. The study also revealed that chat chewers had 2.156 [95\% CI: 1.267-3.670] times the higher risk of developing depressive symptoms as compared to no-chewers, holding effect other covariates fixed.

Holding effect of other covariates constant, the odds of having depression symptoms forthose respondents who had been quarantined for 14 were 2.251 [95\% CI: 1.325-3.823] times greater than those without a quarantine history. Furthermore, depressive symptoms were 3.126 [95\% CI: 1.880-5.197] times more common among those who utilized social media regularly compared to those who did not use regular basis, while keeping other covariates constant.

Respondents who were exposed to COVID-19 had a 3.870 [95\% CI: 1.757-8.527] times higher chance of experiencing depressive symptoms than those who were not. According to the findings of this study, respondents who had family members infected with COVID-19 were 1.925 [95\% CI: 1.153-3.214] times more likely to develop depressive symptoms than those whose family members were not infected with COVID-19. Depressive symptoms were 2.613 [95\% CI: 1.428-4.781] times more common among participants who experienced sleeping issues than among those who did not (Table 2).

\section{Factors Associated with Anxiety Symptoms Among Tepi Town Residents}

In bivariate analysis, gender, alcohol consumption, the habit of chewing Khat, smoking habit, being quarantined for 14 days, frequently using social media, fear of COVID-19, influence on social interaction, taking preventive medicine, sleep problems, family members infected by COVID-19,

Table 2 Outcome of multivariable Binary logistic regression result for Depression

\begin{tabular}{|c|c|c|c|c|c|c|c|}
\hline \multirow[t]{2}{*}{ Variables } & \multirow[t]{2}{*}{ Categories } & \multirow[t]{2}{*}{ B } & \multirow[t]{2}{*}{ S.E } & \multirow[t]{2}{*}{ Sig } & \multirow[t]{2}{*}{$\operatorname{Exp}(B)$} & \multicolumn{2}{|c|}{$95 \%$ C.I. for $\mathrm{EXP}(\mathrm{B})$} \\
\hline & & & & & & Lower & Upper \\
\hline Gender (ref: male) & Female & 1.843 & 0.419 & 0.000 & 6.315 & 2.778 & 14.357 \\
\hline Alcohol consumption (ref: no) & Yes & 0.891 & 0.262 & 0.001 & 2.438 & 1.457 & 4.078 \\
\hline Chewing Khat (ref: no) & Yes & 0.768 & 0.271 & 0.005 & 2.156 & 1.267 & 3.670 \\
\hline Smoking habit (ref: no) & Yes & 0.580 & 0.260 & 0.025 & 1.787 & 1.074 & 2.972 \\
\hline Quarantined for 14 days (ref: no) & Yes & 0.811 & 0.270 & 0.003 & 2.251 & 1.325 & 3.823 \\
\hline Frequently Use social media (ref: no) & Yes & 1.140 & 0.259 & 0.000 & 3.126 & 1.880 & 5.197 \\
\hline Fear COVID-19 (ref: no) & Yes & 0.392 & 0.251 & 0.118 & 1.481 & 0.905 & 2.423 \\
\hline Sleep problems (ref: no) & Yes & 0.961 & 0.308 & 0.002 & 2.613 & 1.428 & 4.781 \\
\hline Family member infected by COVID-19 (ref: no) & Yes & 0.655 & 0.261 & 0.012 & 1.925 & 1.153 & 3.214 \\
\hline Exposed to COVID-19 (ref: no) & Yes & 1.353 & 0.403 & 0.001 & 3.870 & 1.757 & 8.527 \\
\hline \multirow[t]{2}{*}{ HHI (ref: low) } & Moderate & 0.310 & 0.281 & 0.269 & 1.363 & 0.787 & 2.363 \\
\hline & High & -1.095 & 0.693 & 0.114 & 0.334 & 0.086 & 1.300 \\
\hline Nagelkerke R Square & 0.382 & & & & & & \\
\hline Hosmer and Lemeshow test & 0.870 & & & & & & \\
\hline
\end{tabular}

$B$ Coefficient, S.E. standard error, Sig. P value, $\operatorname{Exp}(B)$ odds ratio, C.I. 95\% confidence interval for odds ratio 
and and HHI were found to be significant factors in anxiety symptoms among residences of Tepi town.

Keeping the effect of other covariates fixed, the female residents were 4.591 [95\% CI: 1.627-12.946] times more likely than males to have anxiety symptoms. The odds of developing anxiety symptoms among those who had a history of alcohol usage were 1.797 [95\% CI: 1.052-3.068] times more likely than those who had no alcohol history. The odds of respondents who chew Khat had 2.053 [95\% CI: 1.184-3.558] times more likely to develop anxiety symptoms than those who did not chew Khat. The odds of smokers were 1.883 [95\% CI: 1.112-3.191] times more likely to have anxiety symptoms than non-smokers. In other words, the odds of having anxiety symptoms for the smoker group were $88.3 \%$ higher than that of nonsmokers.

The odds of having anxiety symptoms were 1.902 [95\% CI: 1.098-3.296] times higher among respondents who were quarantined for 14 days compared to respondents without a quarantine history. Participants who used social media frequently were 1.849 [95\% CI: $1.107-3.09$ ] times more likely to have anxiety symptoms as compared to non-users. The odds of experiencing anxiety symptoms for respondents who fear COVID-19 were 1.776 [95\% CI: 1.065-2.963] times higher than those who were not fear COVID-19. According to the findings of this study, respondents whose social interactions were altered by COVID19 had a 3.197 [95\% CI: 1.543-6.623] times higher likelihood of acquiring anxiety than those who did not.

According to the findings of this study, respondents who used traditional preventive medicine had 2.475 [95\% CI: 1.143-5.360] times more likely of getting anxiety symptoms than those who did not. Anxiety symptoms were 2.254 [95\%
CI: 1.246-4.078] times more prevalent in participants who experienced sleeping problems than in persons who did not have such problems. According to the findings of this study, respondents whose family members were infected with COVID-19 were 1.837 [95\% CI: 1.060-3.182] times more likely of having anxiety symptoms than those who did not suffer such problems. The study also found that those with moderate levels of hope had 2.687 [95\% CI: 1.533-4.711] times the chances of having anxious symptoms as compared to those with low levels of hope (Table 3: Shows the oOutcome of a multivariable binary logistic regression for anxiety.).

\section{Factors Associated with Stress Symptoms Among Tepi Town Residents}

According to the result of multivariable logistic regression, gender, the habit of chewing Khat, smoking habits, being quarantined for 14 days, frequently using social media, fear of COVID-19, influence on social interaction, sleep problems, and HHI were found to be significant factors of stress symptoms. However, family members infected by COVID-19, alcohol consumption, and taking preventive medicine were found to be insignificant variables at a 5\% significance level.

By keeping the effect of other covariates constant, the odds of female respondents were 3.155 [95\% CI: 1.324-7.517] times more likely to develop stress symptoms as compared to their male counterparts. Compared to those who do not chew Khat, Khat chewers were 2.110 [95\% CI: 1.318-3.378] times more likely to experience stress symptoms. Smokers had a 1.787 [95\% CI: 1.074-2.972] times higher chance of having stress symptoms than non-smokers.

Table 3 Outcome of a multivariable binary logistic regression for anxiety

\begin{tabular}{|c|c|c|c|c|c|c|c|}
\hline \multirow[t]{2}{*}{ Variables } & \multirow[t]{2}{*}{ Categories } & \multirow[t]{2}{*}{$\mathrm{B}$} & \multirow[t]{2}{*}{ S.E } & \multirow[t]{2}{*}{ Sig } & \multirow[t]{2}{*}{$\operatorname{Exp}(B)$} & \multicolumn{2}{|c|}{ 95\% C.I. for $\operatorname{EXP}(B)$} \\
\hline & & & & & & Lower & Upper \\
\hline Gender (ref: male) & Female & 1.524 & 0.529 & 0.004 & 4.591 & 1.627 & 12.946 \\
\hline Alcohol consumption (ref: no) & Yes & 0.586 & 0.273 & 0.032 & 1.797 & 1.052 & 3.068 \\
\hline Chewing Khat (ref: no) & Yes & 0.719 & 0.281 & 0.010 & 2.053 & 1.184 & 3.558 \\
\hline Smoking habit (ref: no) & Yes & 0.633 & 0.269 & 0.019 & 1.883 & 1.112 & 3.191 \\
\hline Quarantined for 14 days (ref: no) & Yes & 0.643 & 0.281 & 0.022 & 1.902 & 1.098 & 3.296 \\
\hline Frequently use social media (ref: no) & Yes & 0.615 & 0.262 & 0.019 & 1.849 & 1.107 & 3.09 \\
\hline Fear COVID-19 (ref: no) & Yes & 0.574 & 0.261 & 0.028 & 1.776 & 1.065 & 2.963 \\
\hline Influence social interaction (ref: no) & Yes & 1.162 & 0.372 & 0.002 & 3.197 & 1.543 & 6.623 \\
\hline Taking traditional preventive medicine (ref: no) & Yes & 0.906 & 0.394 & 0.022 & 2.475 & 1.143 & 5.360 \\
\hline Sleep problems (ref: no) & Yes & 0.813 & 0.302 & 0.007 & 2.254 & 1.246 & 4.078 \\
\hline Family member infected by COVID-19(ref: no) & Yes & 0.608 & 0.280 & 0.030 & 1.837 & 1.060 & 3.182 \\
\hline \multirow[t]{2}{*}{ Hearth hope index (ref: low) } & Moderate & 1.724 & 0.317 & 0.000 & 5.605 & 3.010 & 10.436 \\
\hline & High & 0.005 & 0.644 & 0.994 & 1.005 & 0.285 & 3.547 \\
\hline Nagelkerke R Square & 0.433 & & & & & & \\
\hline Hosmer and Lemeshow test & 0.614 & & & & & & \\
\hline
\end{tabular}

$B$ coefficient, S.E. standard error, Sig. $P$ value, $\operatorname{Exp}(B)$ odds ratio, C.I. $95 \%$ confidence interval for odds ratio 
The estimated odds of having stress symptoms for those who had been quarantined for 14 days were 1.960 [95\% CI: 1.217-3.156] times higher as compared to those who had no quarantine history. Respondents who used social media frequently were 3.126 [95\% CI: 1.880-5.197] times more likely to have stress symptoms than those who did not use social media frequently.

Furthermore, respondents who feared COVID-19 had 1.835 [95\% CI: 1.176-2.863] times higher odds of stress symptoms than those respondents who did not fear COVID19. According to the findings, people who had their social connections impacted by COVID-19 were 2.069 [95\% CI: 1.095-3.908] times more likely to experience stress symptoms than those who did not. Participants with sleeping issues were 1.721 [95\% CI: 1.029-2.879] times more likely to have stress symptoms than those who did not. Moreover, the study also found that people with moderate levels of hope had a 2.849 [95\% CI: 1.735-4.677] times higher likelihood of acquiring stress symptoms than those with low levels of hope (Table 4: shows the oOutcome of a multivariable binary logistic regression for stress).

\section{Discussion}

This study was conducted to evaluate the symptoms of depression, anxiety, and stress among the residences of Tepi town during the COVID-19 pandemic lockdown. According to current findings, the prevalence of depression, anxiety, and stress symptoms were found to be $37.7 \%, 39.0 \%$, and
$44.2 \%$ respectively among residents of Tepi town. This was a little bit higher than the reports from China among the general population during the epidemic situation, which had depression of $30.3 \%$, anxiety of $36.1 \%$, and stress of $32.1 \%$ [41]. This is also higher than previous study report from Indonesian migrant workers depression $10.15 \%$, anxiety 9.25\%, and stress $2.39 \%$ [42]; in Gondar town, Ethiopia, during the early stages of the COVID-19 pandemic, depression was $32.0 \%$, anxiety was $25.8 \%$, and stress was $14.7 \%$ [43]. Among healthcare workers in Singapore and India during the COVID-19 pandemic, depression was $10.6 \%$, anxiety was $15.7 \%$, and stress was $5.2 \%$ [44]. Another study from China found that $28.8 \%$ of 1210 Chinese individuals had anxiety, $53.8 \%$ had stress, and $16.1 \%$ had depression [45].

However, a study conducted in Iran indicated that the prevalence was $63.4 \%, 42.1 \%$, and $52.1 \%$ of the population, respectively, that had stress, anxiety, and depression symptoms, which was higher than our study findings [46]. Disparities in depression, anxiety, and stress prevalence may be attributed to socioeconomic differences, the impact of COVID-19, culture, ethnicity, and the tool used to assess these mental health outcomes. A previous study also reported that ethnicity differences played an important role in creating an elevated prevalence of depression, anxiety, and stress symptoms among adults during COVID-19 [47]. Elements commonly associated with race or ethnicity, such as socioeconomic level or country of origin, might increase the likelihood of being exposed to these types of mental issues [48, 49].

Gender, alcohol consumption, chewing Khat, smoking habit, quarantine for 14 days, frequent use of social media,

Table 4 Outcome of a multivariable binary logistic regression for stress

\begin{tabular}{|c|c|c|c|c|c|c|c|}
\hline \multirow[t]{2}{*}{ Variables } & \multirow[t]{2}{*}{ Categories } & \multirow[t]{2}{*}{$\mathrm{B}$} & \multirow[t]{2}{*}{ S.E } & \multirow[t]{2}{*}{ Sig } & \multirow[t]{2}{*}{$\operatorname{Exp}(B)$} & \multicolumn{2}{|c|}{$\begin{array}{l}\text { 95\% C.I. for } \\
\operatorname{EXP(B)}\end{array}$} \\
\hline & & & & & & Lower & Upper \\
\hline Gender (ref: male) & Female & 1.149 & 0.443 & 0.010 & 3.155 & 1.324 & 7.517 \\
\hline Alcohol consumption (ref: no) & Yes & 0.097 & 0.235 & 0.679 & 1.102 & 0.695 & 1.748 \\
\hline Chewing Khat (ref: no) & Yes & 0.747 & 0.240 & 0.033 & 2.110 & 1.318 & 3.378 \\
\hline Smoking habit (ref: no) & Yes & 0.580 & 0.260 & 0.025 & 1.787 & 1.074 & 2.972 \\
\hline Quarantined for 14 days (ref: no) & Yes & 0.673 & 0.243 & 0.041 & 1.960 & 1.217 & 3.156 \\
\hline Frequently use social media (ref: no) & Yes & 1.140 & 0.259 & 0.000 & 3.126 & 1.880 & 5.197 \\
\hline Fear COVID-19 (ref: no) & Yes & 0.607 & 0.227 & 0.032 & 1.835 & 1.176 & 2.863 \\
\hline Influence social interaction (ref: no) & Yes & 0.727 & 0.325 & 0.025 & 2.069 & 1.095 & 3.908 \\
\hline Taking preventive medicine (ref: no) & Yes & 0.462 & 0.328 & 0.159 & 1.587 & 0.834 & 3.020 \\
\hline Sleep problems (ref: no) & Yes & 0.543 & 0.263 & 0.039 & 1.721 & 1.029 & 2.879 \\
\hline Family member infected by COVID-19 (ref: no) & Yes & 0.155 & 0.244 & 0.527 & 1.167 & 0.723 & 1.885 \\
\hline \multirow[t]{2}{*}{ Hearth hope index (ref: low) } & Moderate & 1.047 & 0.253 & 0.000 & 2.849 & 1.735 & 4.677 \\
\hline & High & -0.710 & 0.618 & 0.251 & 0.492 & 0.146 & 1.652 \\
\hline Nagelkerke R Square & 0.209 & & & & & & \\
\hline Hosmer and Lemeshow test & 0.398 & & & & & & \\
\hline
\end{tabular}

$B$ Coefficient, S.E. standard error, Sig $P$ value, $\operatorname{Exp}(B)$ odds ratio, C.I. $95 \%$ confidence interval for odds ratio 
sleep problems, family members infected by COVID-19, and exposure to COVID-19 were influential factors for depression. On the other hand, gender, alcohol consumption, chewing Khat, quarantine for 14 days, frequent use of social media, fear of COVID-19, the influence of social interaction, taking traditional preventive medicine, sleep problem, and family member infected by COVID-19 were the main contributing factors to anxiety Chewing Khat, smoking habit, and 14-day quarantine were determining factors associated with stress, as were fear of COVID-19, the influence of social interaction, having sleep problems, and having moderate levels of hope.

When compared to male contemporaries, being a woman raised the risk of depression, anxiety, and stress. This conclusion is consistent with findings from general population studies in Mexico [50] and China [51]. Females may have a higher anxiety prevalence than males due to biological factors such as hormonal fluctuations associated with pregnancy and menstruation [52-54]. Females are more susceptible to depression due to the interaction of genetic, biochemical, hormonal, social, and psychological factors $[15,55]$. The existence of androgen receptors in males may provide [56], and testosterone hormone does not cycle like estrogen in women, which protects guys as well. Furthermore, testosterone is converted to estrogen in the male brain, and estrogen receptors mediate protective effects. The hormone does not cycle in the same way as estrogen does in women, which protects men as well [57].

Furthermore, the disparity in the prevalence of depression, anxiety, and stress between men and women may be due to cultural differences [58]. In Ethiopia, intimate relationship violence against women is more common [59]. Gender-based violence has been demonstrated in prior studies to have a significant influence on women's mental health [60]. In addition, the high prevalence of anxiety and depression among women residents may be due to women's culturally imposed family responsibilities. All of these factors may contribute to a higher prevalence of depression and anxiety in women than in men.

Furthermore, smokers had a higher risk of depression and stress than non-smokers. A study from Ethiopia and Australia backs up this conclusion [15, 61]. This is because smokers are more likely to acquire respiratory issues such as lung cancer and chronic obstructive pulmonary disease (COPD), which exacerbate COVID-19's effects. As a result, they will experience depression and anxiety. Additionally, smoking lowers the dopamine hormone released in the brain, which causes happiness or pleasure [62, 63]. As a result, they are easily depressed, and this provides a clue on how to provide sufficient support for these susceptible people to reduce their burden.

Data from various countries revealed that the COVID-19 outbreak and associated quarantine measures hurt mental health, causing a wide range of psychological problems and increasing symptoms of psychological distress such as anxiety, depression, suicidal ideation, and post-traumatic stress disorder in the general population [1, 5, 9]. In line with this, the findings of this investigation revealed that a 14-day quarantine was linked to greater levels of depression, anxiety, and stress. These findings could be linked to several sociodemographic factors, such as a sense of worry about jobs, money, health, stigma, and isolation. This conclusion was supported by research conducted in Indonesia and China $[14,64]$.

According to the findings of this study, fear of COVID19 was linked to more severe anxiety and stress symptoms among the people of Tepi town. This finding is consistent with a Chinese study [65]. According to a study conducted on medical students, fear of the pandemic, home quarantine, and misinformation distributed on the internet are predicted to exacerbate college students' psychological issues [7, 66]. Moreover, the citizens' dissatisfaction has been worsened by an increase in the number of confirmed illnesses and deaths. As a result, if family members are absent, they may be more concerned about the health of their family members and themselves, leading to increased stress and anxiety symptoms [32].

People who live with a COVID-19 high-risk family member have much greater anxiety levels. Similar to these findings, $75.2 \%$ of Chinese people are concerned about other family members contracting COVID-19 [46, 51]. Close family bonds and concerns about family members may cause anxiety. We found that exposure to COVID-19 has a significant relationship with depression levels. Similar to our results, several studies found that a low perceived risk of developing COVID-19 was strongly associated with a low level of stress and anxiety [46, 51]. Another study found that the perceived risk of COVID-19 infection increased the likelihood of anxiety or depression significantly [67].

People who were exposed to regular COVID-19 news on social media were more likely to be depressed and stressed, but less likely to be nervous, according to this study. It can be caused by erroneous statistical data and rumors that circulate in cyberspace. To avoid inaccurate information and public health statistics, the government and health policymakers must give accurate and up-to-date information. In this context, a study found that having access to current and accurate health information is linked to less stress [46, 51, 68]. However, users must be empowered to efficiently use electronic information.

According to our findings, alcohol consumption and chewing Khat were strongly associated with depression and anxiety symptoms among inhabitants. This study's findings are similar to those of studies conducted in the UK and New Zealand [69, 70]. People with poor mental health are more likely to participate in unhealthy activities 
such as smoking and excessive alcohol intake [71]. This is consistent with previous studies [72, 73]. Substance use, such as alcohol and Khat, typically precedes the symptoms of exhaustion and social difficulties associated with depression and anxiety [74]. As a result, they are easily frightened and sad, and this provides a clue to providing adequate care for these vulnerable people to minimize their load. This finding is supported by a previous study from Ethiopia [43] and Australia [75]. This is also supported by previous studies [73, 76].

According to the findings of this study, sleep problems were linked to more severe anxiety and stress symptoms among people in Tepi town. This finding is in line with previous research done both before and during the pandemic. Before the pandemic, in Saudi Arabia, those who experienced sleep disruption had greater levels of depression, anxiety, and stress [77, 78]. Furthermore, several studies have reported the impact of COVID-19 not only on anxiety and stress levels but also on sleep patterns among individuals [79-81]. Individuals and students who suffer from greater levels of stress and anxiety have reported alterations in sleep patterns and sleep difficulties as a result of emotional discomfort $[81,82]$. Furthermore, a study of Chinese medical students done during the epidemic found a link between sleep disorders, stress, and depression [5]. In addition, Dinis et al. found a link between poor sleep and depression in their review [83]. As we sleep, our body and mind rebuild themselves and get stronger as we sleep. When we have poor sleep, our bodies are unable to relax sufficiently, making us unhappy, depressed, anxious, and stressed.

According to this study, the current pandemic has resulted in social isolation, and altered social interactions, which is harmful to the physical and mental health of society and leads to a higher level of anxiety and stress. This discovery is in line with ones from Mexico and the Gondar town [15, 50]. Having more social interactions was linked to a higher risk of anxiety and stress than having no social engagement. This discovery is in line with ones from Mexico and the Gondar town $[15,50]$. When there were a considerable number of close contacts, this could be due to probable disinformation or information overload [15, 84]. Similarly, closer contact raises the risk of contracting the fatal illness. As a result, precautionary measures such as social separation, frequent hand washing, mask use, and sanitizer are highly suggested.

According to the findings of this study, taking preventive traditional medicine was linked to anxiety symptoms among the people of Tepi town. This could be because those who use traditional preventive medicine are more concerned about uncontrollable side effects. The moderate hearth hope index is also linked with depression, stress, and anxiety symptoms. It might be linked with losing their hope due to the high level of spread and the death-like nature of the diseases.

\section{Limitations and Strengths of the Study}

The current study tried to assess depression, anxiety, and stress symptoms among residents of Tepi town, southwest Ethiopia. This study was not conducted without limitations. Some limitations faced while conducting this study were as follows: (i) In this cross-sectional study, we cannot prove a causal relationship. (ii) The survey questions regarding depression, anxiety, and stress were self-reported, which will contribute to a certain amount of answer bias. (iii) In addition to the variables that we took into account, there may be other factors that are related to the prevalence of depression, anxiety, and stress symptoms among residents that require further study. Despite this limitation, the study is among the very few studies in developing countries in which standardized tools of measurement are used. Moreover, the study considered the timely issue.

\section{Conclusion}

The study results showed that depression, anxiety, and stress symptoms were found to be highly prevalent among Tepi town residents. Being female, chewing Khat, smoking, being quarantined for 14 days, frequently using social media, and having sleeping problems were all found to be significantly associated with an increased risk of developing depression, anxiety, and stress symptoms, whereas alcohol consumption and family members infected by COVID-19 were considerably linked to depression and anxiety symptoms. Fear of COVID-19, influence on social interaction, and having a moderate level of hope were substantially related to stress and anxiety symptoms, while taking preventive medicine was found to be a significant factor in anxiety symptoms among Tepi town residents. Therefore, it is recommended to design intervention strategies to reduce the risk of these problems and to minimize and/or improve the mental health of Tepi town residents.

Abbreviations COVID-19: Corona virus disease 2019; CI: Confidence interval; DASS: Depression, anxiety, and stress symptom; IBM: International business machine; OR: Odds ratio; HHI: Hearth hope index

Acknowledgements We would like to thank the respondents for their kind and timely response during data collection.

Author Contribution SAT \& MEL: Participated in study conception, and design. SAT, MEL \& SSH participated in the data extraction, performing analysis, and interpreting results, and manuscript drafting. AAS, SSJ, BMT, \& TWW revised the manuscript. Finally, all authors read and approved the final manuscript.

Availability of Data and Materials The datasets used in this study are available from the corresponding author on reasonable request. 


\section{Declarations}

Ethics Approval and Consent to Participate Ethical clearance was obtained from the Ethical Review Board of Mizan-Tepi University, formal permission letters were taken from the administration of the Tepi town, and verbal consent was taken from the participants. Personal identity is not used to protect confidentiality.

Consent for Publication Not applicable.

Competing Interests The authors declare no competing interests.

\section{References}

1. Qiu J, Shen B, Zhao M, Wang Z, Xie B, Xu Y. A nationwide survey of psychological distress among Chinese people in the COVID-19 epidemic: implications and policy recommendations. General Psychiatry. 2020;33(2).

2. Caccialanza R, Laviano A, Lobascio F, Montagna E, Bruno R, Ludovisi S, Corsico A, et al. Early nutritional supplementation in non-critically ill patients hospitalized for the 2019 novel coronavirus disease (COVID-19): Rationale and feasibility of a shared pragmatic protocol. Nutrition. 2020;74:110835.

3. Rothe C, Schunk M, Sothmann P, Bretzel G, Froeschl G, Wallrauch $\mathrm{C}$, Zimmer T, et al. Transmission of 2019-nCoV infection from an asymptomatic contact in Germany. New England Journal of Medicine. 2020;382(10):970-1.

4. Wang Y, Di Y, Ye J, Wei W. Study on the public psychological states and its related factors during the outbreak of coronavirus disease 2019 (COVID-19) in some regions of China. Psychol Health Med. 2021;26(1):13-22.

5. Huang Y, Zhao N. "Generalized anxiety disorder, depressive symptoms and sleep quality during COVID-19 outbreak in China: a web-based cross-sectional survey. Psychiatry research. 2020;288:112954.

6. Wasserman D, Iosue M, Wuestefeld A, Carli V. Adaptation of evidence-based suicide prevention strategies during and after the COVID-19 pandemic. World Psychiatry. 2020;19(3):294-306.

7. Brooks SK, et al. The psychological impact of quarantine and how to reduce it: rapid review of the evidence. The lancet. 2020;395(10227):912-20.

8. Li Q, Guan X, Wu P, Wang X, Zhou L, Tong Y, Ren R, et al. Early transmission dynamics in Wuhan, China, of novel coronavirusinfected pneumonia. New England Journal of Medicine. 2020.

9. Brooks S, Webster R, Smith L, Woodland L, Wessely S, Greenberg N, Rubin GJ. The psychological impact of quarantine and how to reduce it: rapid review of the evidence. The Lancet. 2020;395(10227):912-920.

10. Mazza C, Ricci E, Biondi S, Colasanti M, Ferracuti S, Napoli C, Roma P. A nationwide survey of psychological distress among Italian people during the COVID-19 pandemic: immediate psychological responses and associated factors. International Journal of Environmental Research and Public Health. 2020;17(9):3165.

11. Hyland P, Shevlin M, McBride O, Murphy J, Karatzias T, Bentall RP, Martinez A, Vallières F. Anxiety and depression in the Republic of Ireland during the COVID-19 pandemic. Acta Psychiatr Scand. 2020;142(3):249-56.

12. Shigemura J, Ursano RJ, Morganstein JC, Kurosawa M, Benedek DM. Public responses to the novel 2019 coronavirus (2019-nCoV) in Japan: mental health consequences and target populations. Psychiatry Clin Neurosci. 2020;74(4):281.
13. Salari N, et al. Prevalence of stress, anxiety, depression among the general population during the COVID-19 pandemic: a systematic review and meta-analysis. Glob Health. 2020;16(1):1-11.

14. Harjana NPA, Januraga PP, Indrayathi PA, Gesesew HA, Ward PR. Prevalence of depression, anxiety, and stress among repatriated Indonesian migrant workers during the COVID-19 pandemic. Frontiers in Public Health. 2021;9.

15. Akalu TY, et al. Depression, anxiety, and stress symptoms and its associated factors among residents of Gondar Town during the early stage of COVID-19 pandemic. Risk Management and Healthcare Policy. 2021;14:1073.

16. Rodríguez-Rey R, Garrido-Hernansaiz H, Collado S. Psychological impact and associated factors during the initial stage of the coronavirus (COVID-19) pandemic among the general population in Spain. Front Psychol. 2020;11:1540.

17. Dalky HF, Gharaibeh A. Depression, anxiety, and stress among college students in Jordan and their need for mental health services. In Nursing Forum. 2019;54(2):205-12.

18. Lakhan R, Agrawal A, Sharma M. Prevalence of depression, anxiety, and stress during COVID-19 pandemic. Journal of Neurosciences in Rural Practice. 2020.

19. Wang Y, Xu B, Zhao G, Cao R, He X, Fu S. Is quarantine related to immediate negative psychological consequences during the 2009 H1N1 epidemic? Gen Hosp Psychiatry. 2011;33(1):75-7.

20. Rubin GJ, Wessely S. The psychological effects of quarantining a city. BJM. 2020;368.

21. Conversano C, Marchi L, Miniati M. Psychological distress among healthcare professionals involved in the covid19 emergency: vulnerability and resilience factors. Clin Neuropsychiatry. 2020;17(2).

22. Wilkinson RG, Marmot M, (eds). Social determinants of health: the solid facts. World Health Organization, 2003.

23. Marazziti D, Pozza A, Di Giuseppe M, Conversano C. The psychosocial impact of COVID-19 pandemic in Italy: a lesson for mental health prevention in the first severely hit European country. Psychol Trauma Theory Res Pract Policy. 2020;12(5):531.

24 Di Nicola M, et al. Serum 25-hydroxyvitamin D levels and psychological distress symptoms in patients with affective disorders during the COVID-19 pandemic. Psychoneuroendocrinology. 2020;122:104869.

25. WHO World Mental Health Survey Consortium. Prevalence, severity, and unmet need for treatment of mental disorders in the World Health Organization World Mental Health Surveys. JAMA. 2004;291(21):2581-2590.

26. Gilmour H, Patten SB. Depression and work impairment. Health Rep. 2007;18(1):9-22.

27. Arya R, Antonisamy B, Kumar S. Sample size estimation in prevalence studies. The Indian Journal of Pediatrics. 2012;79(11):1482-8.

28. Osman A, Wong JL, Bagge CL, Freedenthal S, Gutierrez PM, Lozano G. The depression anxiety stress Scales-21 (DASS-21): further examination of dimensions, scale reliability, and correlates. J Clin Psychol. 2012;68(12):1322-38.

29. Damanik ED, "The measurement of reliability, validity, items analysis and normative data of Depression Anxiety Stress Scale (DASS)," Diunduh dari, 2011.

30. Lovibond PF, Lovibond SH. The structure of negative emotional states: comparison of the depression anxiety stress scales (DASS) with the Beck depression and anxiety inventories. Behav Res Ther. 1995;33(3):335-43.

31. Buysse DJ, Reynolds III CF, Monk TH, Berman SR, Kupfer DJ. The pittsburgh sleep quality index: a new instrument for psychiatric practice and research. Psychiatry Res. 1989;28(2):193-213.

32. Escobar DFSS, de Jesus TF, Noll M. Family and school context: effects on the mental health of Brazilian students. 
International Journal of Environmental Research and Public Health 2020;17(17):6042.

33. Zhong Q-Y, Gelaye B, Sánchez SE, Williams MA. Psychometric properties of the Pittsburgh Sleep Quality Index (PSQI) in a cohort of Peruvian pregnant women. J Clin Sleep Med. 2015;11(8):869-877.

34. Bigalke JA, Greenlund IM, Carter JR. Sex differences in selfreport anxiety and sleep quality during COVID-19 stay-at-home orders. Biol Sex Differ. 2020;11(1):1-11.

35. Kafle K, Shrestha DB, Baniya A, Lamichhane S, Shahi M, Gurung B, Tandan P, Ghimire A, Budhathoki P. Psychological distress among health service providers during COVID-19 pandemic in Nepal. PloS One. 2021;16(2):e0246784.

36. Kaye Herth PhD, R. N. Development and refinement of an instrument to measure hope. Research and Theory for Nursing Practice. 1991;5(1):39.

37. Li L-R et al. "Effects of intrinsic and extrinsic factors on the level of hope and psychological health status of patients with cervical cancer during radiotherapy. 2017;23: 3508.

38. Hosmer DW, Hosmer T, Le Cessie S, Lemeshow S. A comparison of goodness-of-fit tests for the logistic regression model. Stat Med. 1997;16(9):965-80.

39. Radwan E, Radwan A, Radwan W, Pandey D. Perceived Stress among School Students in Distance Learning During the COVID19 Pandemic in the Gaza Strip, Palestine. Augmented Human Research. 2021;6(1):1-13.

40. Parthiban K, Pandey D, Pandey BK. Impact of SARS-CoV-2 in Online Education, Predicting and Contrasting Mental Stress of Young Students: A Machine Learning Approach. Augmented Human Research. 2021;6(1):1-7.

41. Wang C, Pan R, Wan X, Tan Y, Linkang X, Ho CS, Ho RC. Immediate psychological responses and associated factors during the initial stage of the 2019 coronavirus disease (COVID19) epidemic among the general population in China. International Journal of Environmental Research and Public Health. 2020;17(5):1729.

42. Harjana NPA, Januraga PP, Indrayathi PA, Gesesew HA, Ward PR. Prevalence of depression, anxiety, and stress among repatriated Indonesian migrant workers during the COVID-19 pandemic. Frontiers in Public Health. 2021;9:442.

43. Akalu TY, Gelaye KA, Bishaw MA, Tilahun SY, Yeshaw Y, Azale T, Tsegaye T, Asmelash D, Yonas Akalu Y. Depression, anxiety, and stress symptoms and its associated factors among residents of Gondar Town during the early stage of COVID-19 pandemic. Risk Manag Healthc Policy. 2021;14:1073.

44. Meslie Y, Enbeyle W, Pandey BK, Pramanik S, Pandey D, Dadeech P, ... Saini A (2021). Machine intelligence-based trend analysis of COVID-19 for total daily confirmed cases in Asia and Africa. In Methodologies and Applications of Computational Statistics for Machine Intelligence (pp. 164-185). IGI Global.

45. Wang $\mathrm{C}$, et al. A longitudinal study on the mental health of general population during the COVID-19 epidemic in China. Brain Behav Immun. 2020;87:40-8.

46. Khademian F, Delavari S, Koohjani Z, Khademian Z. An investigation of depression, anxiety, and stress and its relating factors during COVID-19 pandemic in Iran. BMC Public Health. 2021;21(1):1-7.

47. McKnight-Eily LR, Okoro CA, Strine TW, Verlenden J, Hollis ND, Njai R, Mitchell EW, Board A, Puddy R, Thomas C. Racial and ethnic disparities in the prevalence of stress and worry, mental health conditions, and increased substance use among adults during the COVID-19 pandemic-United States, April and May 2020. Morbidity and Mortality Weekly Report. 2021;70(5):162.

48. General USPHSOotS, Services CfMH, Health NIoM, Abuse USS, Administration MHS, Mental health: culture, race, and ethnicity: a supplement to mental health: A report of the Surgeon General.
Department of Health and Human Services, US Public Health Service, 2001.

49. Pandey D, Islam T, Malik MA. Novel coronavirus disease (SARSCOV-2): an overview. Asian Journal of Advances in Medical Science. $2021 ; 8-12$

50. Cortés-Álvarez NY, Piñeiro-Lamas R, Vuelvas-Olmos CR. Psychological effects and associated factors of COVID-19 in a Mexican sample. Disaster Med Public Health Prep. 2020;14(3):413-24.

51. Wang $\mathrm{C}$, et al. Immediate psychological responses and associated factors during the initial stage of the 2019 coronavirus disease (COVID-19) epidemic among the general population in China. Int J Environ Res Public Health. 2020;17(5):1729.

52. Rizvi N. Frequency of depression and anxiety among tuberculosis patients. Journal of Tuberculosis Research. 2016;4(04):183.

53. Pradesh H, Pradesh H, Pradesh HJEJPMR, "Assessment of the frequency of depression and anxiety among tuberculosis patients at a tertiary care health centre a cross. 2018; 5(4):496-499.

54. Kashif M, Javed MK, Pandey D. A surge in cyber-crime during COVID-19. Indonesian Journal of Social and Environmental Issues (IJSEI). 2020;1(2):48-52.

55. Albert PR. Why is depression more prevalent in women? Journal of psychiatry \& neuroscience: JPN. 2015;40(4):219.

56. McEwen BS, Milner TA. Hippocampal formation: shedding light on the influence of sex and stress on the brain. Brain Res Rev. 2007;55(2):343-55.

57. Gillies GE, McArthur S. Acciones de estrógenos en el cerebro y la base para la acción diferencial en hombres y mujeres: un caso para medicamentos específicos para el sexo. Revisiones farmacológicas junio de. 2010;62(2):155-98.

58. Parkerson HA, Thibodeau MA, Brandt CP, Zvolensky MJ, Asmundson GJJJoAD, "Cultural-based biases of the GAD-7. 2015;31: 38-42.

59. Abeya SG, Afework MF, Yalew AW. Intimate partner violence against women in west Ethiopia: a qualitative study on attitudes, woman's response, and suggested measures as perceived by community members. Reproductive Health. 2012;9(1):1-11.

60. Gelaye B, Arnold D, Williams MA, Goshu M, Berhane Y. Depressive symptoms among female college students experiencing gender-based violence in Awassa, Ethiopia. J Interpers Violence. 2009;24(3):464-481.

61. Hamdi N, et al. Implementation of a multi-component school lunch environmental change intervention to improve child fruit and vegetable intake: a mixed-methods study. Int J Environ Res Public Health. 2020;17(11):3971.

62. Hickie I, et al. Reduced hippocampal volumes and memory loss in patients with early-and late-onset depression. Br J Psychiatry. 2005;186(3): 197-202.

63. Boden JM, Fergusson DM, Horwood LJ. Cigarette smoking and depression: tests of causal linkages using a longitudinal birth cohort. Br J Psychiatry. 2010;196(6):440-6.

64. Kassaw C, Pandey D. COVID-19 pandemic related to anxiety disorder among communities using public transport at Addis Ababa, Ethiopia, March 2020: Cross-sectional Study Design. Human Arenas. 2021;1-10.

65. Yu M, Tian F, Cui Q, Wu H. Prevalence and its associated factors of depressive symptoms among Chinese college students during the COVID-19 pandemic. BMC Psychiatry. 2021;21(1):1-8.

66. Mackolil J, Mackolil JJAjop "Addressing psychosocial problems associated with the COVID-19 lockdown. 2020; 51: 102156.

67. Shevlin M, McBride O, Murphy J, Miller JB, Hartman TK, Levita L, Mason L, et al. Anxiety, depression, traumatic stress and COVID-19-related anxiety in the UK general population during the COVID-19 pandemic. BJPsych Open. 2020;6(6).

68. Kassaw C. The magnitude of psychological problem and associated factor in response to COVID-19 pandemic among communities living in Addis Ababa, Ethiopia, March 2020: A 
Cross-Sectional Study Design. Psychol Res Behav Manag. 2020;13:631-40.

69. Jia R, et al. "Mental health in the UK during the COVID-19 pandemic: cross-sectional analyses from a community cohort study. BMJ open. 2020;10(9):e040620.

70 Gasteiger N, et al. Depression, anxiety and stress during the COVID-19 pandemic: results from a New Zealand cohort study on mental well-being. BMJ open. 2021;11(5):e045325.

71. Lipari R, Van Horn S. Smoking and mental illness among adults in the United States. The CBHSQ report. 2017.

72. Ridner SL, Staten RR, Danner FW. Smoking and depressive symptoms in a college population. J Sch Nurs. 2005;21(4):229-35.

73. Pedrelli P, Farabaugh AH, Zisook S, Tucker D, Rooney K, Katz J, Clain AJ, Petersen TJ, Fava M. Gender, depressive symptoms and patterns of alcohol use among college students. Psychopathology. 2011;44(1):27-33.

74. Boden JM, Fergusson DM, Horwood LJ. Cigarette smoking and depression: tests of causal linkages using a longitudinal birth cohort. Br J Psychiatry. 2010;196(6):440-6.

75. Hamdi N, Ellison B, McCaffrey J, Metcalfe JJ, Hoffman A, Haywood P, Prescott MP. Implementation of a multi-component school lunch environmental change intervention to improve child fruit and vegetable intake: a mixed-methods study. International Journal of Environmental Research and Public Health. 2020;17(11):3971.

76. Wang C-J, Yang T-F, Wang G-S, Zhao Y-Y, Yang L-J, Bi B-N. Association between dietary patterns and depressive symptoms among middle-aged adults in China in 2016-2017. Psychiatry Res. 2018;260:123-129.

77. Ahammed B, Khan B, Jahan N, Shohel TA, Hossain T, Islam N. Determinants of generalized anxiety, depression, and subjective sleep quality among university students during COVID-19 pandemic in Bangladesh. Dr. Sulaiman Al Habib Medical Journal. 2021;3(1):27-35.

78. Al-Maddah EM, Al-Dabal BK, Khalil MS. Prevalence of sleep deprivation and relation with depressive symptoms among medical residents in King Fahd University Hospital, Saudi Arabia. Sultan Qaboos Univ Med J. 2015;15(1):e78.

79. Khongsai L, Anal TSC, Rapheileng AS, Kh TS, Shah MK, Pandey D. Combating the spread of COVID-19 through community participation. Global Social Welfare. 2021;8(2):127-32.

80. Adhikari SP, et al. Epidemiology, causes, clinical manifestation and diagnosis, prevention and control of coronavirus disease (COVID-19) during the early outbreak period: a scoping review. Infect Dis Poverty. 2020;9(1):1-12.

81. Cellini N, Canale N, Mioni G, Costa S. Changes in sleep pattern, sense of time and digital media use during COVID-19 lockdown in Italy. J sleep res. 2020;29(4):e13074.

82. Marelli S, et al. Impact of COVID-19 lockdown on sleep quality in university students and administration staff. J Neurol. 2021;268(1):8-15.

83. Pandey D, Ogunmola GA, Enbeyle W, Abdullahi M, Pandey BK, Pramanik S. COVID-19: A framework for effective delivering of online classes during lockdown. Human Arenas.2021;1-15.

84. Burtscher J, Burtscher M, Millet GP. (Indoor) isolation, stress and physical inactivity: vicious circles accelerated by Covid-19? Scandinavian Journal of Medicine \& Science in Sports. 2020.

Publisher's Note Springer Nature remains neutral with regard to jurisdictional claims in published maps and institutional affiliations.

\section{Authors and Affiliations}

\section{Seid Ali Tareke ${ }^{1}$. Mesfin Esayas Lelisho ${ }^{1}$ (1) . Sali Suleman Hassen ${ }^{1}$ - Adem Aragaw Seid ${ }^{1}$. Sebwedin Surur Jemal ${ }^{1}$. Belete Mulatu Teshale ${ }^{1} \cdot$ Teramaj Wongel Wotale $^{2} \cdot$ Binay Kumar Pandey $^{3}$}

\author{
Seid Ali Tareke \\ aliseid1a@gmail.com \\ Sali Suleman Hassen \\ salisuleman20@gmail.com \\ Adem Aragaw Seid \\ ademgita@gmail.com \\ Sebwedin Surur Jemal \\ sebwedinsurur@gmail.com \\ Belete Mulatu Teshale \\ alimulatu@gmail.com \\ Teramaj Wongel Wotale \\ teramaj24@gmail.com
}

Binay Kumar Pandey

binaydece@gmail.com

1 Department of Statistics, College of Natural and Computational Science, Mizan-Tepi University, P.O. Box 121, Tepi, Ethiopia

2 Department of Statistics, College of Natural Science, Mettu University, Mettu, Oromia, Ethiopia

3 Department of Information Technology, College of Technology, Govind Ballabh Pant University of Agriculture and Technology Pantnagar, U S Nagar Uttarakhand, India 